\title{
Harmonic Spinors on a Family of Einstein Manifolds
}

\author{
Guido Franchetti \\ Dipartimento di Matematica Giuseppe Peano \\ Università degli Studi di Torino \\ 10123 Torino, Italy. \\ guido.franchetti@unito.it
}

\begin{abstract}
The purpose of this paper is to study harmonic spinors defined on a 1-parameter family of Einstein manifolds which includes Taub-NUT, Eguchi-Hanson and $P^{2}(\mathbb{C})$ with the Fubini-Study metric as particular cases. We discuss the existence of and explicitly solve for spinors harmonic with respect to the Dirac operator twisted by a geometrically preferred connection. The metrics examined are defined, for generic values of the parameter, on a non-compact manifold with the topology of $\mathbb{C}^{2}$ and extend to $P^{2}(\mathbb{C})$ as edge-cone metrics. As a consequence, the subtle boundary conditions of the Atiyah-Patodi-Singer index theorem need to be carefully considered in order to show agreement between the index of the twisted Dirac operator and the result obtained by counting the explicit solutions.
\end{abstract}

\section{Introduction}

Einstein 4-manifolds play an important rôle in mathematical physics. They arise as Euclidean continuations of solutions of Einstein equations in general relativity [16, 17], as moduli space of solitons [25, 11], as particle models in the geometric models of matter framework [7, 15]. Particularly important examples are the Taub-NUT (TN), EguchiHanson (EH), Atiyah-Hitchin (AH) manifolds and the Fubini-Study (FS) metric on $P^{2}(\mathbb{C})$.

Interestingly, two known 1-parameter families of Einstein metrics interpolate between these examples. Both families are initially defined on a non-compact manifold, but admit a conformal compactification as metrics with an edge-cone singularity along an embedded 2-surface. Both are half-conformally-flat, have non-negative scalar curvature and are rotationally symmetric, that is, admit an isometric $S O(3)$ or $S U(2)$ action. The first family, introduced in [22], is defined on $S^{4} \backslash P^{2}(\mathbb{R})$ and extends to a family of edgecone metrics of positive scalar curvature on $S^{4}$ with cone angle $\frac{2 \pi}{k-2}$ along $P^{2}(\mathbb{R})$. The parameter $k$ is an integer $\geq 3$ For $k=3$ one obtains the smooth round metric on the 4 -sphere. For $k=4$ the metric admits a double cover isometric to $P^{2}(\mathbb{C})$ with the FS metric. For $k \rightarrow \infty$ the metric converges to the AH metric.

The second family was introduced in [1]. It depends on a real parameter $\beta \in[0,2]$, has positive scalar curvature for $\beta \in(0,2)$ and, for $\beta \neq 0$, extends to a 1-parameter family of edge-cone metrics on $P^{2}(\mathbb{C})$ with cone angle $2 \pi \beta$ along an embedded $S^{2}$. For 
$\beta=1$ one obtains the smooth FS metric. For $\beta=2$, a $\mathbb{Z}_{2}$ quotient of the metric gives the Ricci-flat EH metric on $T S^{2}$. For $\beta \rightarrow 0$ one obtains the Ricci-flat TN metric.

Harmonic spinors are solutions of the twisted (massless) Dirac equation $D_{\mathcal{A}} \psi=0$. From the mathematical viewpoint, studying the spectrum of Dirac operators and in particular whether or not they admit zero eigenvalues, and therefore harmonic spinors, is an interesting problem both per se and in connection with index theorems $[23,8,10$, $9,5]$.

In the physical literature, solutions of the Dirac equation are known as (fermionic) zero modes and twisting of the Dirac equation is often called minimal coupling (to a gauge field). Fermionic zero modes on a non-trivial background have been studied e.g. in connection with fractionalisation of fermionic charge [26], non-perturbative effects in supersymmetric gauge theories [35, 3, 2], superconducting cosmic strings [37].

In this paper we study harmonic spinors defined on the second family of Einstein manifolds described above. From the physical viewpoint, we are concerned with fermionic zero modes on an Einstein background coupled to an Abelian gauge field. In particular, we will see that the kernel of the non-twisted Dirac operator is trivial. However, the manifolds admit a geometrically preferred 2-form which is closed, self-dual (hence harmonic), $L^{2}$ and rotationally invariant. For $\beta=0, \beta=2$ this 2 -form is the (up to scale) unique harmonic $L^{2}$ 2-form $\mathcal{F}$ on TN and EH [24], predicted by Sen's $S$-duality conjecture [36], while for $\beta=1$ it becomes the Kähler form of the FS metric. Considered on the compactified manifold, which has the topology of $P^{2}(\mathbb{C}), \mathcal{F}$ generates the middle dimension cohomology group and corresponds to the Poincaré dual of the non-trivial 2-cycle.

Interpreting $\mathcal{F}$ as a curvature form, we consider the kernel of the Dirac operator twisted by the corresponding Abelian connection. In this case, non-trivial left-handed harmonic spinors exist. In fact, we show that the kernel of the Dirac operator decomposes as a direct sum of all the irreducible $S U(2)$ representations up to a dimension determined by the spinor charge and the parameter $\beta$. Thanks to the rotational symmetry of the problem, the harmonic condition reduces to an ODE which can be integrated, so we explicitly obtain all the solutions.

With all the solutions at hand, we can compute the index of the twisted Dirac operator and compare it with the result obtained via the Atiyah-Patodi-Singer (APS) index theorem [8]. In order to avoid dealing with the edge-cone singularity, we work on the non-compact manifold with boundary obtained by truncating our space at some finite radius $r_{0}$ and then take the limit $r_{0} \rightarrow \infty$. In typical applications of the APS theorem to non-compact spaces [33, 27, 28], the manifold under consideration has infinite volume. Square-integrable spinors need then to decay asymptotically and the subtle boundary conditions of the APS index theorem are automatically satisfied. Instead, the space we consider has, for $2 \neq \beta \neq 0$, finite volume and agreement between counting of the explicit solutions and the APS index theorem is only recovered once boundary conditions are taken into proper account.

The paper is structured as follows. In Section 2 we recall some basic facts about 
Bianchi IX spaces, that is 4-manifolds admitting an isometric $S U(2)$ action with (generically) 3-dimensional orbits. We then write down the twisted Dirac operator on a generic Bianchi IX space, generalising previous work [27] in that we do not assume the curvature tensor to be (anti) self-dual. In Section 3 we introduce the 1-parameter family of Einstein metrics [1], following the presentation recently given in [6], and review some of its properties. In Section 4 we show how, as a consequence of Lichnerowicz's formula, the non-twisted Dirac operator admits no non-trivial harmonic spinors, and discuss twisting by the geometrically preferred connection with curvature $\mathcal{F}$. In Section 5 the various ingredients come together as we explicitly solve for harmonic spinors, comment on their properties, and compare the results with those obtained via the APS index theorem.

\section{The Twisted Dirac Operator on Bianchi IX Spaces}

\subsection{Metric and Connection Form}

In terms of the left-invariant 1-forms on $S U(2)$,

$$
\begin{aligned}
& \eta_{1}=\sin \psi \mathrm{d} \theta-\cos \psi \sin \theta \mathrm{d} \phi, \\
& \eta_{2}=\cos \psi \mathrm{d} \theta+\sin \psi \sin \theta \mathrm{d} \phi, \\
& \eta_{3}=\mathrm{d} \psi+\cos \theta \mathrm{d} \phi,
\end{aligned}
$$

for $\theta \in[0, \pi], \phi \in[0,2 \pi), \psi \in[0,4 \pi)$, a Bianchi IX metric has the form

$$
g_{\mathrm{IX}}=f^{2} \mathrm{~d} r^{2}+a^{2} \eta_{1}^{2}+b^{2} \eta_{2}^{2}+c^{2} \eta_{3}^{2}
$$

with $a, b, c, f$ functions of the transverse coordinate $r$ only.

The right (respectively left) action of $S U(2)$ on itself, generated by the left-invariant vector fields $\left\{X_{i}\right\}$ (right-invariant vector fields $\left\{Z_{i}\right\}$ ), given in Appendix B, induces a right (left) action on a Bianchi IX space. The left $S U(2)$ action is an action by isometries. A Bianchi IX metric for which $a=b$, known as bi-axial, admits an additional $U(1)$ isometry group, generated by the left-invariant Killing vector field $X_{3}=\partial / \partial \psi$, and given by translation along the circles parametrised by $\psi$. The generic orbit of the right $S U(2)$ action on a Bianchi IX space has the topology of $S^{3}$. By restricting $\psi$ to $[0,2 \pi)$ in (1) one obtains the left-invariant 1-forms on $S O(3)$ and the generic orbit of the corresponding right $S O(3)$ action on a Bianchi IX space has the topology of $P^{3}(\mathbb{R})$.

For the time being we make no further assumption on $g_{\mathrm{IX}}$. We define the proper radius $R$ by $\mathrm{d} R=f \mathrm{~d} r$, denote differentiation with respect to $r$ by a prime ', and with respect to $R$ by a dot . We take the orthonormal coframe

$$
e^{1}=a \eta_{1}, \quad e^{2}=b \eta_{2}, \quad e^{3}=c \eta_{3}, \quad e^{4}=-f \mathrm{~d} r=-\mathrm{d} R .
$$

In our conventions Latin indices vary in the range $\{1,2,3\}$ and Greek indices vary in the range $\{1,2,3,4\}$. The Einstein summation convention is enforced but, since we are 
working with an orthonormal coframe, we do not distinguish upper indices from lower ones. We denote the orthonormal frame dual to $\left\{e^{\mu}\right\}$ by $\left\{E_{\mu}\right\}$. Note that

$$
E_{1}=a^{-1} X_{1}, \quad E_{2}=b^{-1} X_{2}, \quad E_{3}=c^{-1} X_{3}, \quad E_{4}=-f^{-1} \partial_{r}=-\partial / \partial R,
$$

where

$$
\begin{aligned}
& X_{1}=\sin \psi \partial_{\theta}+\frac{\cos \psi}{\sin \theta}\left(\cos \theta \partial_{\psi}-\partial_{\phi}\right), \\
& X_{2}=\cos \psi \partial_{\theta}-\frac{\sin \psi}{\sin \theta}\left(\cos \theta \partial_{\psi}-\partial_{\phi}\right), \\
& X_{3}=\partial_{\psi}
\end{aligned}
$$

are the left-invariant vector fields on $S U(2)$ dual to the forms $\left\{\eta_{i}\right\}$.

Defining the conformally invariant quantities

$$
A=\frac{b^{2}+c^{2}-a^{2}}{2 b c}, \quad B=\frac{c^{2}+a^{2}-b^{2}}{2 c a}, \quad C=\frac{a^{2}+b^{2}-c^{2}}{2 a b},
$$

the non-vanishing components of the Levi-Civita connection form $\omega$ are given by

$$
\begin{aligned}
& \omega_{12}=-C \eta_{3}, \quad \omega_{23}=-A \eta_{1}, \quad \omega_{31}=-B \eta_{2}, \\
& \omega_{34}=-\dot{c} \eta_{3}, \quad \omega_{14}=-\dot{a} \eta_{1}, \quad \omega_{24}=-\dot{b} \eta_{2} .
\end{aligned}
$$

The corresponding curvature form $\Omega$ is

$$
\begin{aligned}
& \Omega_{12}=-\dot{C} \mathrm{~d} R \wedge \eta_{3}+(C-\dot{a} \dot{b}-A B) \eta_{1} \wedge \eta_{2}, \\
& \Omega_{23}=-\dot{A} \mathrm{~d} R \wedge \eta_{1}+(A-\dot{b} \dot{c}-B C) \eta_{2} \wedge \eta_{3}, \\
& \Omega_{31}=-\dot{B} \mathrm{~d} R \wedge \eta_{2}+(B-\dot{c} \dot{a}-C A) \eta_{3} \wedge \eta_{1}, \\
& \Omega_{14}=-\ddot{a} \mathrm{~d} R \wedge \eta_{1}+(\dot{a}-\dot{b} C-\dot{c} B) \eta_{2} \wedge \eta_{3}, \\
& \Omega_{24}=-\ddot{b} \mathrm{~d} R \wedge \eta_{2}+(\dot{b}-\dot{c} A-\dot{a} C) \eta_{3} \wedge \eta_{1}, \\
& \Omega_{34}=-\ddot{c} \mathrm{~d} R \wedge \eta_{3}+(\dot{c}-\dot{a} B-\dot{b} A) \eta_{1} \wedge \eta_{2} .
\end{aligned}
$$

\subsection{Spherically Symmetric Self-dual 2-forms}

Knowledge of the spherically symmetric self-dual 2-forms on a Bianchi IX space will be needed in Section 4 where we discuss twisting of the Dirac operator. Any spherically symmetric 2-form on a Bianchi IX space is locally of the form $\mathrm{d} \mathcal{A}$, with

$$
\mathcal{A}=\mathcal{A}_{i} \eta_{i}, \quad \mathcal{A}_{i}=\mathcal{A}\left(X_{i}\right) .
$$

Since

$$
\mathrm{d} \mathcal{A}=\frac{\dot{\mathcal{A}}_{1}}{a} e^{1} \wedge e^{4}-\frac{\mathcal{A}_{1}}{b c} e^{2} \wedge e^{3}+\frac{\dot{\mathcal{A}}_{2}}{b} e^{2} \wedge e^{4}-\frac{\mathcal{A}_{2}}{c a} e^{3} \wedge e^{1}+\frac{\dot{\mathcal{A}}_{3}}{c} e^{3} \wedge e^{4}-\frac{\mathcal{A}_{3}}{a b} e^{1} \wedge e^{2},
$$


$\mathrm{d} \mathcal{A}$ is self-dual with respect to the volume form

$$
\mathrm{vol}=e^{1} \wedge e^{2} \wedge e^{3} \wedge e^{4}=f a b c \mathrm{~d} r \wedge \eta_{1} \wedge \eta_{2} \wedge \eta_{3}=f a b c \sin \theta \mathrm{d} r \wedge \mathrm{d} \psi \wedge \mathrm{d} \theta \wedge \mathrm{d} \psi
$$

if and only if

$$
\dot{\mathcal{A}}_{1}=-\frac{a}{b c} \mathcal{A}_{1}, \quad \dot{\mathcal{A}}_{2}=-\frac{b}{c a} \mathcal{A}_{2}, \quad \dot{\mathcal{A}}_{3}=-\frac{c}{a b} \mathcal{A}_{3} .
$$

Clearly, the functions $\mathcal{A}_{i}$ are determined up to a multiplicative constant.

\subsection{The Dirac Operator}

Let $\left\{\gamma_{\mu}\right\}, \mu=1, \ldots, 4$, be Clifford generators,

$$
\gamma_{\mu} \gamma_{\nu}+\gamma_{\nu} \gamma_{\mu}=-2 \delta_{\mu \nu} I_{4}
$$

where $I_{4}$ is the $4 \times 4$ identity matrix. We take the generators in the chiral form

$$
\gamma_{a}=\left(\begin{array}{cc}
\mathbf{0} & \boldsymbol{\sigma}_{a} \\
-\boldsymbol{\sigma}_{a} & \mathbf{0}
\end{array}\right), a=1,2,3, \quad \gamma_{4}=\left(\begin{array}{cc}
\mathbf{0} & -i \mathbf{1} \\
-i \mathbf{1} & \mathbf{0}
\end{array}\right)
$$

where $\mathbf{1}, \mathbf{0}$ are the $2 \times 2$ identity and null matrices and $\left\{\boldsymbol{\sigma}_{a}\right\}$ the Pauli matrices. Dirac spinors which are eigenvectors of the chirality operator $-\gamma_{1} \gamma_{2} \gamma_{3} \gamma_{4}$ with eigenvalue +1 (respectively -1) are called left-handed (right-handed). In the chiral representation (15), the third and fourth (first and second) components of a left-handed (right-handed) Dirac spinor vanish.

The twisted Dirac operator $D_{\mathcal{A}}$ associated to the orthonormal coframe $\left\{e^{\mu}\right\}$, its dual frame $\left\{E_{\mu}\right\}$ and the Abelian real-valued connection $\mathcal{A}$ is, see e.g. [30],

$$
\not D_{\mathcal{A}}=\gamma_{\mu}\left[\left(E_{\mu}+i \mathcal{A}\left(E_{\mu}\right)\right) I_{4}-\frac{1}{8}\left[\gamma_{\rho}, \gamma_{\sigma}\right] \omega_{\rho \sigma}\left(E_{\mu}\right)\right] \text {. }
$$

The non-twisted Dirac operator $\not D$ is obtained by setting $\mathcal{A}=0$.

We rewrite (16) in the form

$$
\not D_{\mathcal{A}}=\gamma_{4}\left[\left(E_{4}+i \mathcal{A}\left(E_{4}\right)+\frac{1}{2} \omega_{k 4}\left(E_{k}\right)\right) I_{4}+\left(\begin{array}{cc}
-\mathbf{1} & \mathbf{0} \\
\mathbf{0} & \mathbf{1}
\end{array}\right) \mathbf{B}_{\mathcal{A}}\right]
$$

in order to isolate the contribution of the Dirac operator $\mathbf{B}_{\mathcal{A}}$ induced by $\not D_{\mathcal{A}}$ on a hypersurface of large radius [19]. Making use of the commutation relations

$$
\left[\gamma_{i}, \gamma_{j}\right]=-2 i \epsilon_{i j k}\left(\begin{array}{cc}
\boldsymbol{\sigma}_{k} & \mathbf{0} \\
\mathbf{0} & \boldsymbol{\sigma}_{k}
\end{array}\right), \quad\left[\gamma_{4}, \gamma_{i}\right]=2 i\left(\begin{array}{cc}
\boldsymbol{\sigma}_{i} & \mathbf{0} \\
\mathbf{0} & -\boldsymbol{\sigma}_{i}
\end{array}\right)
$$

we calculate

$$
-\frac{1}{8}\left[\gamma_{\rho}, \gamma_{\sigma}\right] \omega_{\rho \sigma}\left(E_{\mu}\right)=\left(\begin{array}{cc}
\mathbf{1} & \mathbf{0} \\
\mathbf{0} & \mathbf{1}
\end{array}\right) \frac{i}{4} \epsilon_{i j k} \omega_{i j}\left(E_{\mu}\right) \boldsymbol{\sigma}_{k}+\left(\begin{array}{cc}
\mathbf{1} & \mathbf{0} \\
\mathbf{0} & -\mathbf{1}
\end{array}\right) \frac{i}{2} \omega_{k 4}\left(E_{\mu}\right) \boldsymbol{\sigma}_{k} .
$$


Using (7), (8) and the relations

$$
\gamma_{a}=i \sigma_{a} \gamma_{4}\left(\begin{array}{cc}
-\mathbf{1} & \mathbf{0} \\
\mathbf{0} & \mathbf{1}
\end{array}\right), \quad \gamma_{\mu}\left(\begin{array}{cc}
\mathbf{1} & \mathbf{0} \\
\mathbf{0} & -\mathbf{1}
\end{array}\right) \frac{i}{2} \omega_{k 4}\left(E_{\mu}\right) \sigma_{k}=\frac{1}{2} \gamma_{4} \omega_{k 4}\left(E_{k}\right),
$$

we rewrite (16) as

$$
\begin{aligned}
\not D_{\mathcal{A}}=\gamma_{4}\left\{\left[E_{4}+i \mathcal{A}\left(E_{4}\right)+\frac{1}{2} \omega_{k 4}\left(E_{k}\right)\right] I_{4}+\right. \\
\left.+\left[i \sigma_{a}\left(E_{a}+i \mathcal{A}\left(E_{a}\right)\right)-\frac{1}{4} \epsilon_{i j k} \omega_{i j}\left(E_{a}\right) \sigma_{a} \sigma_{k}\right]\left(\begin{array}{cc}
-\mathbf{1} & \mathbf{0} \\
\mathbf{0} & \mathbf{1}
\end{array}\right)\right\} .
\end{aligned}
$$

Therefore,

$$
\mathbf{B}_{\mathcal{A}}=i \sigma_{a}\left(E_{a}+i \mathcal{A}\left(E_{a}\right)\right)-\frac{1}{4} \epsilon_{i j k} \omega_{i j}\left(E_{a}\right) \sigma_{a} \sigma_{k}
$$

Since

$$
-\omega_{k 4}\left(E_{k}\right)=\frac{\dot{a}}{a}+\frac{\dot{b}}{b}+\frac{\dot{c}}{c}, \quad-\frac{1}{2} \epsilon_{i j k} \omega_{i j}\left(E_{a}\right) \sigma_{a} \sigma_{k}=\frac{A}{a}+\frac{B}{b}+\frac{C}{c},
$$

setting

$$
D_{i}=X_{i}+i \mathcal{A}\left(X_{i}\right)
$$

we get

$$
\begin{aligned}
& \not D_{\mathcal{A}}=\gamma_{4}\left\{\left[-\frac{\partial_{r}}{f}+i \mathcal{A}\left(E_{4}\right)-\frac{1}{2}\left(\frac{\dot{a}}{a}+\frac{\dot{b}}{b}+\frac{\dot{c}}{c}\right)\right] I_{4}+\left(\begin{array}{cc}
-\mathbf{1} & \mathbf{0} \\
\mathbf{0} & \mathbf{1}
\end{array}\right) \mathbf{B}_{\mathcal{A}}\right\}, \\
& \mathbf{B}_{\mathcal{A}}=i\left(\frac{\boldsymbol{\sigma}_{1} D_{1}}{a}+\frac{\boldsymbol{\sigma}_{2} D_{2}}{b}+\frac{\boldsymbol{\sigma}_{3} D_{3}}{c}\right)+\frac{\mathbf{1}}{2}\left(\frac{A}{a}+\frac{B}{b}+\frac{C}{c}\right) .
\end{aligned}
$$

Note that the operator $\not_{\mathcal{A}}$ has the form

$$
\not D_{\mathcal{A}}=\left(\begin{array}{cc}
\mathbf{0} & \mathbf{T}_{\mathcal{A}}^{\dagger} \\
\mathbf{T}_{\mathcal{A}} & \mathbf{0}
\end{array}\right)
$$

with

$$
\begin{aligned}
\mathbf{T}_{\mathcal{A}} & =\left[\frac{i \partial_{r}}{f}+\mathcal{A}\left(E_{4}\right)+\frac{i}{2}\left(\frac{\dot{a}}{a}+\frac{\dot{b}}{b}+\frac{\dot{c}}{c}\right)\right] \mathbf{1}+i \mathbf{B}_{\mathcal{A}} \\
& =\left[\frac{i \partial_{r}}{f}+\mathcal{A}\left(E_{4}\right)+\frac{i}{2}\left(\frac{\dot{a}+A}{a}+\frac{\dot{b}+B}{b}+\frac{\dot{c}+C}{c}\right)\right] \mathbf{1}-\frac{\boldsymbol{\sigma}_{1} D_{1}}{a}-\frac{\boldsymbol{\sigma}_{2} D_{2}}{b}-\frac{\boldsymbol{\sigma}_{3} D_{3}}{c} \\
\mathbf{T}_{\mathcal{A}}^{\dagger} & =\left[\frac{i \partial_{r}}{f}+\mathcal{A}\left(E_{4}\right)+\frac{i}{2}\left(\frac{\dot{a}}{a}+\frac{\dot{b}}{b}+\frac{\dot{c}}{c}\right)\right] \mathbf{1}-i \mathbf{B}_{\mathcal{A}} \\
& =\left[\frac{i \partial_{r}}{f}+\mathcal{A}\left(E_{4}\right)+\frac{i}{2}\left(\frac{\dot{a}-A}{a}+\frac{\dot{b}-B}{b}+\frac{\dot{c}-C}{c}\right)\right] \mathbf{1}+\frac{\boldsymbol{\sigma}_{1} D_{1}}{a}+\frac{\boldsymbol{\sigma}_{2} D_{2}}{b}+\frac{\boldsymbol{\sigma}_{3} D_{3}}{c}
\end{aligned}
$$


The operator $\mathbf{T}_{\mathcal{A}}^{\dagger}$ is the formal adjoint of $\mathbf{T}_{\mathcal{A}}$. In fact, let $\psi, \phi$ be Dirac spinors. Their $L^{2}$ product induced by the volume element (12) is

$$
\langle\phi, \psi\rangle=\int \phi^{\dagger} \psi \text { vol, }
$$

where $^{\dagger}$ denotes the transpose conjugate. Integrating by parts and discarding boundary terms we get

$$
\begin{aligned}
\left\langle\phi, \frac{i \partial_{r} \psi}{f}\right\rangle & =i \int \phi^{\dagger} \frac{\partial_{r} \psi}{f} f a b c \mathrm{~d} r \wedge \eta_{1} \wedge \eta_{2} \wedge \eta_{3} \\
& =-i \int\left[\frac{\partial_{r} \phi^{\dagger}}{f} \psi+\phi^{\dagger}\left(\frac{\dot{a}}{a}+\frac{\dot{b}}{b}+\frac{\dot{c}}{c}\right) \psi\right] f a b c \mathrm{~d} r \wedge \eta_{1} \wedge \eta_{2} \wedge \eta_{3} \\
& =\left\langle\frac{i \partial_{r} \phi}{f}+i \phi\left(\frac{\dot{a}}{a}+\frac{\dot{b}}{b}+\frac{\dot{c}}{c}\right), \psi\right\rangle .
\end{aligned}
$$

Moreover, up to boundary terms,

$$
\begin{aligned}
\left\langle\phi, X_{1} \psi\right\rangle & =\int X_{1}\left(\phi^{\dagger} \psi f a b c\right) \mathrm{d} r \wedge \eta_{1} \wedge \eta_{2} \wedge \eta_{3}-\int X_{1}\left(\phi^{\dagger}\right) \psi f a b c \mathrm{~d} r \wedge \eta_{1} \wedge \eta_{2} \wedge \eta_{3} \\
& =-\int X_{1}\left(\phi^{\dagger}\right) \psi f a b c \mathrm{~d} r \wedge \eta_{1} \wedge \eta_{2} \wedge \eta_{3}=-\left\langle X_{1} \phi, \psi\right\rangle,
\end{aligned}
$$

having used the relation, valid for any function $h$,

$$
X_{a}(h) \eta_{a} \wedge \mathrm{d} r \wedge \eta_{2} \wedge \eta_{3}=\mathrm{d}\left(h \mathrm{~d} r \wedge \eta_{i} \wedge \eta_{j}\right) .
$$

Hence $\left\langle\phi, \boldsymbol{\sigma}_{1} D_{1} \psi\right\rangle=-\left\langle\boldsymbol{\sigma}_{1} D_{1} \phi, \psi\right\rangle$ and similarly for the terms in $D_{2}, D_{3}$. Therefore, the formal adjoint of $\mathbf{T}_{\mathcal{A}}$ is indeed (29).

\section{A 1-parameter Family of Einstein Metrics}

In Section 5 we will explicitly solve for harmonic spinors on a 1-parameter family of halfconformally-flat Einstein metrics initially found in [1] which includes, for special values of the parameter, celebrated metrics such as the Fubini-Study (FS), Eguchi-Hanson (EH) and Taub-NUT (TN) metrics. Recently, this family has been obtained by conformal rescaling of a hyperbolic analogue of the TN space [6]. We recall the construction here.

Consider the metric, introduced in [31],

$$
g_{\mathrm{hTN}}=V g_{H_{L}^{3}}+V^{-1} \eta_{3}^{2}
$$

Here $g_{H_{L}^{3}}$ is the metric on hyperbolic 3 -space of sectional curvature $-1 /\left(4 L^{2}\right)$,

$$
g_{H_{L}^{3}}=\mathrm{d} r^{2}+4 L^{2} \sinh ^{2}(r / 2 L)\left(\eta_{1}^{2}+\eta_{2}^{2}\right),
$$


$V$ is the positive function

$$
V=\frac{1}{L}\left(\frac{1}{\beta}+\frac{1}{\mathrm{e}^{r / L}-1}\right)=\frac{\mathrm{e}^{r / L}+\beta-1}{\beta L\left(\mathrm{e}^{r / L}-1\right)},
$$

$\beta$ is a constant. The angles have range $\theta \in[0, \pi], \phi \in[0,2 \pi), \psi \in[0,4 \pi)$, while $r \in[0, \infty)$. The apparent singularity at $r=0$ is a coordinate singularity, the topology is that of $\mathbb{C}^{2}$.

The geometry described by (34) is very similar to that of TN. The metrics of both spaces are based on the Gibbons-Hawking ansatz and are of bi-axial Bianchi IX form. The origin is a fixed point of the $U(1)$ action generated by the Killing vector field $X_{3}$, and the space with the origin removed is a circle bundle over a 3-manifold. While for TN the base of the circle bundle is Euclidean space, for (34) is hyperbolic space. In both cases, the topology is that of $\mathbb{C}^{2}$. I will refer to $\mathbb{C}^{2}$ with the metric (34) as hyperbolic Taub-NUT (hTN).

It should be pointed out that there are also important differences between $\mathrm{TN}$ and hTN: TN is a hyperkähler space, hence half-conformally-flat and Ricci-flat, while hTN is half-conformally-flat but not Einstein. Its scalar curvature is given by

$$
s_{\mathrm{hTN}}=-\frac{3}{2 L^{2} V} \text {. }
$$

A 1-parameter family of Einstein metrics can be obtained by conformally rescaling $g_{\mathrm{hTN}}[6]:$ For

$$
\Lambda=\sqrt{\frac{4 L}{\beta}} \frac{1}{(2-\beta) \cosh (r / 2 L)+\beta \sinh (r / 2 L)},
$$

the metric

$$
g_{\beta}=\Lambda^{2} g_{\mathrm{hTN}}
$$

is Einstein with Einstein constant

$$
C_{\beta}=\frac{3}{2} \frac{\beta^{2}}{4 L^{2}}(2-\beta) .
$$

In order for $g_{\beta}$ to be well-defined for all $r \geq 0$, it is necessary to have $\beta \in(0,2]$.

Note that for $\beta=1$, making the substitution

$$
1+\frac{v^{2}}{16 L^{2}}=\exp \left(\frac{r}{L}\right)
$$

we obtain

$$
g_{1}=\lim _{\beta \rightarrow 1} g_{\beta}=\frac{\mathrm{d} v^{2}}{\left(1+\frac{v^{2}}{16 L^{2}}\right)^{2}}+\frac{v^{2}}{4\left(1+\frac{v^{2}}{16 L^{2}}\right)^{2}} \eta_{3}^{2}+\frac{v^{2}}{4\left(1+\frac{v^{2}}{16 L^{2}}\right)}\left(\eta_{1}^{2}+\eta_{2}^{2}\right) .
$$

This is the FS metric on $P^{2}(\mathbb{C}) \backslash P^{1}(\mathbb{C})$ with Einstein constant $3 /\left(8 L^{2}\right)$ [18]. The removed $P^{1}(\mathbb{C})$ corresponds to the asymptotic 2 -sphere $S_{\infty}^{2}$ at $v \rightarrow \infty$. 
The limiting cases $\beta=2$ and $\beta=0$ are particularly interesting. For $\beta \rightarrow 2$, making the substitution

$$
r=2 L \operatorname{arcoth}\left(\frac{w^{2}}{4 L^{2}}\right)
$$

we get

$$
g_{2}=\lim _{\beta \rightarrow 2} g_{\beta}=\frac{\mathrm{d} w^{2}}{1-\left(\frac{2 L}{w}\right)^{4}}+\frac{w^{2}}{4}\left(1-\left(\frac{2 L}{w}\right)^{4}\right) \eta_{3}^{2}+\frac{w^{2}}{4}\left(\eta_{1}^{2}+\eta_{2}^{2}\right) .
$$

This would be the metric on the EH space if $\psi$ had range $[0,2 \pi)$ rather than $[0,4 \pi)$ as in (44). It is therefore a metric on the branched double cover of the EH space and has a conical singularity of excess angle $2 \pi$ along the 2 -sphere $w=2 L$.

The EH metric is usually defined on a space which is topologically the tangent bundle $T S^{2}$ of $S^{2}$. In terms of the coordinates used in (44) the zero section of $T S^{2}$ is obtained for $w=2 L$. The double cover of $T S^{2}$ is homeomorphic to $P^{2}(\mathbb{C}) \backslash\{p\}$ [6]. Adding a point and removing the zero section we obtain the space $\mathbb{C}^{2}$ over which $g_{2}$ is defined. In terms of the coordinate transformation (43), the zero section of $T S^{2}$ has been pushed to $r \rightarrow \infty$, while $w \rightarrow \infty$ corresponds to the point $r=0$.

For $\beta \rightarrow 0$, making the substitution

$$
r=\beta u,
$$

we obtain

$$
g_{0}=\left.\lim _{\beta \rightarrow 0} g_{\beta}\right|_{r=\beta u}=\left(1+\frac{L}{u}\right)\left(\mathrm{d} u^{2}+u^{2}\left(\eta_{1}^{2}+\eta_{2}^{2}\right)\right)+L^{2}\left(1+\frac{L}{u}\right)^{-1} \eta_{3}^{2},
$$

the metric on the TN space.

Asymptotically, setting $\mu=\beta \exp \left(-\frac{r}{2 L}\right)$,

$$
g_{\beta}=\frac{4 L^{2}}{\beta^{2}}\left[\left(\eta_{1}^{2}+\eta_{2}^{2}\right)\left(1+\frac{\mu^{2}(3 \beta-4)}{\beta^{2}}\right)+\mu^{2}\left(\frac{\mathrm{d} r^{2}}{\beta^{2} L^{2}}+\eta_{3}^{2}\right)+O\left(\mu^{3}\right)\right] .
$$

For $\beta \neq 0$ a hypersurface of fixed $r$, which for $r>0$ has the topology of a 3-sphere, collapses in the limit $r \rightarrow \infty$ to the 2-sphere $S_{\infty}^{2}$, the asymptotic boundary of $M_{\beta}$.

The metric $g_{\mathrm{hTN}}$ is defined on a space with the topology of $\mathbb{C}^{2}$. For $\beta \in(0,2]$, the conformally rescaled metric $g_{\beta}$ extends to $P^{2}(\mathbb{C})=\mathbb{C}^{2} \cup P^{1}(\mathbb{C})$, where the added $P^{1}(\mathbb{C})$ is the asymptotic 2 -sphere $S_{\infty}^{2}$ which has self-intersection number one in $P^{2}(\mathbb{C})$. For $\beta \neq 1$, the metric $g_{\beta}$ has an edge cone singularity of deficit/excess angle $2 \pi|1-\beta|$ along $S_{\infty}^{2}[6]$. From now on, for $\beta \in[0,2]$ we denote the space $\mathbb{C}^{2}$ with the metric $g_{\beta}$ by $M_{\beta}$, and, for $\beta \in(0,2]$, its compactification by $P_{\beta}^{2}(\mathbb{C})$.

For the specific values of $a, b, c, f$ of $g_{\mathrm{hTN}}$,

$$
a_{\mathrm{hTN}}=b_{\mathrm{hTN}}=2 L \sqrt{V} \sinh (r / 2 L), \quad c_{\mathrm{hTN}}=1 / \sqrt{V}, \quad f_{\mathrm{hTN}}=-\sqrt{V},
$$


the volume element (12) becomes

$$
\begin{aligned}
\operatorname{vol}_{\beta} & =-\Lambda^{4} a_{\mathrm{hTN}}^{2} \mathrm{~d} r \wedge \eta_{1} \wedge \eta_{2} \wedge \eta_{3}, \\
\Lambda^{4} a_{\mathrm{hTN}}^{2} & =\frac{16 L^{3}}{\beta^{3}} \frac{\exp (r / L)(\exp (r / L)-1)(\exp (r / L)+\beta-1)}{(\exp (r / L)-\beta+1)^{4}} .
\end{aligned}
$$

It can be checked that for $\beta \neq 0,2, M_{\beta}$ has finite volume, given by

$$
\int_{M_{\beta}} \operatorname{vol}_{\beta}=-16 \pi^{2} \int_{\infty}^{0} \Lambda^{4} a_{\mathrm{hTN}}^{2} \mathrm{~d} r=\frac{128 L^{4} \pi^{2}}{3} \frac{(4-\beta)}{\beta^{3}(\beta-2)^{2}},
$$

where we integrate from $\infty$ to 0 since $-\Lambda^{4} a_{\mathrm{hTN}}^{2}<0$. For $\beta=2$ the volume of $M_{\beta}$ diverges. The divergent contribution comes from $r=0$, the portion of EH space usually at infinity brought to finite distance by the transformation (43). In the limit $\beta \rightarrow 0$

$$
\left.\lim _{\beta \rightarrow 0} \operatorname{vol}_{\beta}\right|_{r \rightarrow \beta u}=-L u^{2}\left(1+\frac{L}{u}\right) \mathrm{d} u \wedge \eta_{1} \wedge \eta_{2} \wedge \eta_{3},
$$

hence, as expected, the volume of TN also diverges.

\section{Twisting the Dirac Operator}

The space $M_{\beta}$ admits no non-trivial harmonic spinors. In fact, by Lichnerowicz's formula, see e.g. [30], the square of the Dirac operator $\not D$ is given by

$$
\not D^{2}=\triangle \psi+\frac{s}{4} \psi
$$

where $s$ is the scalar curvature of the manifold, $\triangle=\nabla \circ \nabla$ is the connection Laplacian and $\nabla$ the covariant derivative associated to the spin connection $\omega$. Suppose $\psi$ is an $L^{2}$ harmonic spinor on $M_{\beta}$, (52) then gives

$$
0=\langle\not D \psi, \not D \psi\rangle=\langle\nabla \psi, \nabla \psi\rangle+\frac{s}{4}\langle\psi, \psi\rangle .
$$

For $\beta \in(0,2)$ the scalar curvature $s=4 C_{\beta}$ of $M_{\beta}$ is strictly positive, see (40), hence the non-twisted Dirac operator $\not D$ does not admit non-trivial harmonic spinors. For $\beta=0$, $\beta=2, s=0$ but $M_{\beta}$ has infinite volume. Since a non-compact manifold of infinite volume admits no $L^{2}$ covariantly constant spinors, the conclusion is unchanged.

Consider now $D_{\mathcal{A}}$, the Dirac operator twisted by a connection $\mathcal{A}$ with curvature $\mathcal{F}=\mathrm{d} \mathcal{A}$, and the corresponding generalised Lichnerowicz's formula,

$$
\not D_{\mathcal{A}}^{2} \psi=\triangle \psi+\frac{s}{4} \psi+\frac{1}{2} \mathcal{F}_{\mu \nu} \gamma_{\mu} \gamma_{\nu} \psi
$$

If $\mathcal{F}$ is self-dual, the last term of (54) vanishes when $\psi$ is a right-handed spinor. Hence $M_{\beta}$ admits no non-trivial $L^{2}$ right-handed harmonic spinors, that is, the kernel of $\mathbf{T}_{\mathcal{A}}^{\dagger}$ is trivial. 
As we have just seen, it is necessary to twist the Dirac operator in order to have non-trivial harmonic spinors. To get an interesting problem, the connection $\mathcal{A}$ by which we twist $\not D$ should not be arbitrary, but related to the geometry of $M_{\beta}$. Since $M_{\beta}$ is homeomorphic to $\mathbb{C}^{2}$, its second de Rham cohomology group is trivial. However, as $\not_{\mathcal{A}}$ involves the metric $g_{\beta}$, harmonic, spherically symmetric $L^{2}$ forms are natural candidates.

The easiest way to find harmonic forms on $M_{\beta}$ is to look for closed self-dual forms. Self-duality is a conformally invariant condition, so self-dual forms on hTN are also self-dual on $M_{\beta}$. From (13), (48) we find

$$
\begin{aligned}
& \mathcal{A}_{1}=\mathcal{A}_{2}=\exp \left(\frac{r}{\beta L}(1-\beta)\right)\left(\mathrm{e}^{r / L}-1\right), \\
& \mathcal{A}_{3}=1-\frac{\beta}{\mathrm{e}^{r / L}+\beta-1}=\frac{c_{\mathrm{hTN}}^{2}}{\beta L} .
\end{aligned}
$$

The curvatures $\mathrm{d} \mathcal{A}_{1}, \mathrm{~d} \mathcal{A}_{2}$ are not $L^{2}$ with respect to $g_{\mathrm{hTN}}$, hence, by the conformal invariance of the Hodge operator in middle dimension, they are not $L^{2}$ with respect to $g_{\beta}$ either. Instead, the self-dual 2-form

$$
\mathcal{F}_{3}=\mathrm{d} \mathcal{A}_{3}=\frac{\beta}{L} \frac{\exp (r / L) \mathrm{d} r \wedge \eta_{3}}{(\exp (r / L)+\beta-1)^{2}}-\frac{(\exp (r / L)-1) \eta_{1} \wedge \eta_{2}}{\exp (r / L)+\beta-1}
$$

is $L^{2}$ and exact, but not necessarily $L^{2}$-exact as $\mathcal{A}_{3}$ is not $L^{2}$ with respect to either $g_{\mathrm{hTN}}$ or $g_{\beta}$.

It is interesting to look at what $\mathcal{F}_{3}$ becomes in the special cases $\beta=0,1,2$. For $\beta=1$, making the substitution (41), we have

$$
\left.\mathcal{F}_{3}\right|_{\beta=1}=\frac{1}{8 L^{2}}\left[\frac{v}{\left(1+\frac{v^{2}}{16 L^{2}}\right)^{2}} \mathrm{~d} v \wedge \eta_{3}-\frac{v^{2}}{2\left(1+\frac{v^{2}}{16 L^{2}}\right)} \eta_{1} \wedge \eta_{2}\right] .
$$

This is the Kähler form of the FS metric, and the unique (up to a multiplicative constant) harmonic 2-form on $P^{2}(\mathbb{C})$.

For $\beta=2$, making the substitution (43), we have

$$
\left.\mathcal{F}_{3}\right|_{\beta=2}=-\frac{4 L^{2}}{w^{2}}\left(\frac{2}{w} \mathrm{~d} w \wedge \eta_{3}+\eta_{1} \wedge \eta_{2}\right) .
$$

This 2 -form is invariant under $\psi \rightarrow \psi+2 \pi$, hence descends to the unique $L^{2}$ harmonic form on $\mathrm{EH}[24]$.

In the limit $\beta \rightarrow 0$, making the substitution (45), we have

$$
\left.\lim _{\beta \rightarrow 0} \mathcal{F}_{3}\right|_{r=\beta u}=-\frac{u}{u+L} \eta^{1} \wedge \eta^{2}+\frac{L}{(L+u)^{2}} \mathrm{~d} u \wedge \eta^{3}=\mathrm{d}\left(\frac{u}{u+L} \eta^{3}\right) .
$$

This is the unique (up to a multiplicative constant) $L^{2}$ harmonic 2 -form on TN [24]. We conjecture that $\mathcal{F}_{3}$ is the unique $L^{2}$ harmonic form on $M_{\beta}$ for all $\beta \in[0,2]$. 
Since $M_{\beta}$ is topologically trivial, the Abelian connection $\mathcal{A}$ takes value in the Lie algebra of $\mathbb{R}$, rather than that of $U(1)$. Consider now, for $\beta \in(0,2]$, the conformal compactification $P_{\beta}^{2}(\mathbb{C})$ of $M_{\beta}$. The form $\mathcal{F}_{3}$ is now topologically non-trivial: Since the self-intersection number of $S_{\infty}^{2}$ in $P^{2}(\mathbb{C})$ is one [6] and

$$
\int_{S_{\infty}^{2}} \mathcal{F}_{3}=-4 \pi
$$

$-\mathcal{F}_{3} /(4 \pi)$ is the Poincaré dual of the boundary 2 -sphere $S_{\infty}^{2}$. For $p \in \mathbb{Z}, \frac{i p}{2} \mathcal{A}_{3} \eta_{3}$ can be interpreted as a $U(1)$ connection with first Chern number $-p$.

In the case $\beta=0$, the TN metric $g_{0}$ does not extend to $P^{2}(\mathbb{C})$. Instead, $P^{2}(\mathbb{C})$ arises as the Hausel-Hunsicker-Mazzeo compactification of TN, a topological compactification arising in the study of the harmonic $L^{2}$ cohomology of gravitational instantons [20, 14].

In the next Section, we will explicitly find harmonic spinors for the Dirac operator on $M_{\beta}$ twisted by the connection

$$
\mathcal{A}=\frac{p}{2} \mathcal{A}_{3} \eta_{3}=\frac{p}{2} \frac{\eta_{3}}{\beta L V}
$$

where $p \in \mathbb{R}$ is a constant having the physical interpretation of the spinor charge.

\section{Harmonic Spinors}

We are now going to explicitly solve the equation $\not D_{\mathcal{A}} \psi=0$ on $M_{\beta}$ for $\mathcal{A}$ given by (62).

Since $g_{\beta}$ is bi-axial, that is $a=b \Rightarrow A=B$, and only $\mathcal{A}\left(E_{3}\right) \neq 0$, (28), (29) become

$$
\begin{aligned}
& \mathbf{T}_{\mathcal{A}}=i\left[\left(\frac{\partial_{r}}{f}+\frac{\dot{a}}{a}+\frac{\dot{c}}{2 c}\right) \mathbf{1}+\mathbf{B}_{\mathcal{A}}\right], \\
& \mathbf{T}_{\mathcal{A}}^{\dagger}=i\left[\left(\frac{\partial_{r}}{f}+\frac{\dot{a}}{a}+\frac{\dot{c}}{2 c}\right) \mathbf{1}-\mathbf{B}_{\mathcal{A}}\right], \\
& \mathbf{B}_{\mathcal{A}}=i\left(\begin{array}{cc}
D_{3} / c & X_{-} / a \\
X_{+} / a & -D_{3} / c
\end{array}\right)+\left(\frac{A}{a}+\frac{C}{2 c}\right) \mathbf{1}, \quad \text { where } X_{ \pm}=X_{1} \pm i X_{2} .
\end{aligned}
$$

We now substitute

$$
\begin{aligned}
a & =\Lambda a_{\mathrm{hTN}}, \quad c=\Lambda c_{\mathrm{hTN}}, \quad f=\Lambda f_{\mathrm{hTN}}, \quad A=\frac{c_{\mathrm{hTN}}}{2 a_{\mathrm{hTN}}}, \quad C=1-\frac{c_{\mathrm{hTN}}^{2}}{2 a_{\mathrm{hTN}}^{2}}, \\
D_{3} & =X_{3}+i \mathcal{A}\left(X_{3}\right)=X_{3}+\frac{i p}{2} \frac{1}{\beta L V},
\end{aligned}
$$

where $a_{\mathrm{hTN}}, c_{\mathrm{hTN}}, f_{\mathrm{hTN}}$ are given by (48) and $\Lambda$ by (38). Setting

$$
\lambda=\left[2 L V \sinh \left(\frac{r}{2 L}\right)\right]^{-1}, \quad \tilde{p}=p(\beta L V)^{-1},
$$

we obtain $\mathbf{B}_{\mathcal{A}}=(\sqrt{V} \lambda / 2 \Lambda) \mathbf{P}_{\mathcal{A}}$, with

$$
\mathbf{P}_{\mathcal{A}}=\left(\begin{array}{cc}
\lambda^{-1}\left(2 i X_{3}-\tilde{p}\right) & 2 i X_{-} \\
2 i X_{+} & -\lambda^{-1}\left(2 i X_{3}-\tilde{p}\right)
\end{array}\right)+\left(\frac{\lambda^{2}+2}{2 \lambda}\right) \mathbf{1} .
$$




\subsection{The Dirac Operator on the Squashed 3-sphere}

It is worth pausing to consider the eigenvectors of $\mathbf{P}_{\mathcal{A}}$, which will be needed in Section 5.3 to calculate the index of $\not D_{\mathcal{A}}$ via the APS index theorem. The operator $\mathbf{P}_{\mathcal{A}}$ is essentially the twisted Dirac operator on the squashed 3-sphere, which has been considered in [34]. Its non-twisted version, which can be obtained setting $\tilde{p}=0$, has been studied in [23]. Other useful references include [32, 27, 28]. In order to make the paper self-contained, we give a short treatment here.

Because of the spherical symmetry of the problem, the operators $\not D_{\mathcal{A}}, \mathbf{P}_{\mathcal{A}}$ commute with the scalar Laplacian on the round 3-sphere

$$
\triangle_{S^{3}}=-\left(X_{1}^{2}+X_{2}^{2}+X_{3}^{2}\right)
$$

hence we can restrict $\mathbf{P}_{\mathcal{A}}$ to an eigenspace of $\triangle_{S^{3}}$. The eigenvectors of $\triangle_{S_{3}}$ are given by the irreducible representations $V_{j} \otimes V_{j}$ of $\mathfrak{s l}(2, \mathbb{C}) \oplus \mathfrak{s l}(2, \mathbb{C})$, where $2 j \in \mathbb{Z}$ and $V_{j}$ is the irreducible representation of $\mathfrak{s l}(2, \mathbb{C})$ of dimension $2 j+1$. We use the shorthand notation $\left|j, m, m^{\prime}\right\rangle$ for the element $|j, m\rangle \otimes\left|j, m^{\prime}\right\rangle \in V_{j} \otimes V_{j}$. Let

$$
\left\{\left|j, m, m^{\prime}\right\rangle: 2 m \in \mathbb{Z}, 2 m^{\prime} \in \mathbb{Z},-j \leq m \leq j,-j \leq m^{\prime} \leq j\right\}
$$

be a basis of $V_{j} \otimes V_{j}$ consisting of simultaneous eigenvectors of $\triangle_{S_{3}}$ and $i X_{3}$. The action of $\triangle_{S^{3}}, i X_{3}, i X_{ \pm}$on $\left|j, m, m^{\prime}\right\rangle$ is given by

$$
\begin{aligned}
& \triangle_{S^{3}}\left|j, m, m^{\prime}\right\rangle=j(j+1)\left|j, m, m^{\prime}\right\rangle, \quad i X_{3}\left|j, m, m^{\prime}\right\rangle=m\left|j, m, m^{\prime}\right\rangle, \\
& i X_{+}\left|j, m, m^{\prime}\right\rangle= \begin{cases}\sqrt{(j-m)(j+m+1)}\left|j, m+1, m^{\prime}\right\rangle & -j \leq m<j, \\
0 & m=j,\end{cases} \\
& i X_{-}\left|j, m, m^{\prime}\right\rangle= \begin{cases}\sqrt{(j+m)(j-m+1)}\left|j, m-1, m^{\prime}\right\rangle & -j<m \leq j, \\
0 & m=-j .\end{cases}
\end{aligned}
$$

Take the ansatz $v=\left(C_{1}\left|j, m, m^{\prime}\right\rangle, C_{2}\left|j, m+1, m^{\prime}\right\rangle\right)^{T}$, with $C_{1}, C_{2}$ constants. Acting with $\mathbf{P}_{\mathcal{A}}$ on $v$ we find, for $-j \leq m \leq j-1$,

$$
\left(\begin{array}{c}
{\left[\frac{2 C_{2}}{C_{1}} \sqrt{(j-m)(j+m+1)}+\frac{1}{2 \lambda}\left(2(2 m-\tilde{p})+2+\lambda^{2}\right)\right] C_{1}\left|j, m, m^{\prime}\right\rangle} \\
{\left[\frac{2 C_{1}}{C_{2}} \sqrt{(j-m)(j+m+1)}+\frac{1}{2 \lambda}\left(-2(2 m+2-\tilde{p})+\lambda^{2}+2\right)\right] C_{2}\left|j, m+1, m^{\prime}\right\rangle}
\end{array}\right),
$$

hence $v$ is an eigenvector provided that

$$
\frac{C_{1}}{C_{2}}=\frac{1}{\lambda}\left[\frac{2 m+1-\tilde{p} \pm \sqrt{(2 m+1-\tilde{p})^{2}+4 \lambda^{2}(j-m)(j+m+1)}}{2 \sqrt{(j-m)(j+m+1)}}\right] .
$$

Therefore, for $-j \leq m \leq j-1, \mathbf{P}_{\mathcal{A}}$ has eigenvalues

$$
\frac{\lambda}{2} \pm \frac{1}{\lambda} \sqrt{(2 m+1-\tilde{p})^{2}+4 \lambda^{2}(j-m)(j+m+1)} .
$$


For $m=j$,

$$
\mathbf{P}_{\mathcal{A}}\left(\begin{array}{c}
\left|j, j, m^{\prime}\right\rangle \\
0
\end{array}\right)=\left[\frac{2 j-\tilde{p}}{\lambda}+\frac{\lambda^{2}+2}{2 \lambda}\right]\left(\begin{array}{c}
\left|j, j, m^{\prime}\right\rangle \\
0
\end{array}\right)
$$

hence the eigenvalue is

$$
\frac{2 j+1-\tilde{p}}{\lambda}+\frac{\lambda}{2}
$$

For $m=-j-1$,

$$
\mathbf{P}_{\mathcal{A}}\left(\begin{array}{c}
0 \\
\left|j,-j, m^{\prime}\right\rangle
\end{array}\right)=\left[\frac{2 j+\tilde{p}}{\lambda}+\frac{\lambda^{2}+2}{2 \lambda}\right]\left(\begin{array}{c}
0 \\
\left|j,-j, m^{\prime}\right\rangle
\end{array}\right) .
$$

In summary, the eigenvectors and eigenvalues of $\mathbf{P}_{\mathcal{A}}$ are

\section{Eigenvector}

\section{Eigenvalue}

$$
\begin{array}{cc}
\left(\begin{array}{c}
C_{1}\left|j, m, m^{\prime}\right\rangle \\
C_{2}\left|j, m+1, m^{\prime}\right\rangle
\end{array}\right) & \frac{\lambda}{2} \pm \frac{1}{\lambda} \sqrt{(2 m+1-\tilde{p})^{2}+4 \lambda^{2}(j-m)(j+m+1)},-j \leq m \leq j-1 \\
\left(\begin{array}{c}
0 \\
\left|j,-j, m^{\prime}\right\rangle
\end{array}\right) & \frac{\lambda}{2}+\frac{1}{\lambda}(2 j+1+\tilde{p}), m=-j-1 \\
\left(\begin{array}{c}
\left|j, j, m^{\prime}\right\rangle \\
0
\end{array}\right) & \frac{\lambda}{2}+\frac{1}{\lambda}(2 j+1-\tilde{p}), m=j
\end{array}
$$

with $C_{1} / C_{2}$ satisfying (73). All the eigenvalues have multiplicity $2 j+1$ coming from the "right" $\mathfrak{s l}(2, \mathbb{C})$ representation which has been hidden in the notation.

To examine the large $r$ behaviour of the eigenvalues of $\mathbf{P}_{\mathcal{A}}$ we set

$$
\mu=\beta \exp \left(-\frac{r}{2 L}\right) .
$$

Since $\lambda=\mu+O\left(\mu^{3}\right), \tilde{p}=p+O\left(\mu^{2}\right)$, we obtain

Eigenvector

$\left(\begin{array}{c}\tilde{C}_{1}\left|j, m, m^{\prime}\right\rangle \\ \tilde{C}_{2}\left|j, m+1, m^{\prime}\right\rangle\end{array}\right) \quad \pm \frac{1}{\mu}|2 m+1-p|+O(\mu),-j \leq m \leq j-1, p \neq 2 m+1$

$$
\pm 2 \sqrt{(j-m)(j+m+1)}+O(\mu),-j \leq m \leq j-1, p=2 m+1
$$

$\left(\begin{array}{c}0 \\ \left|j,-j, m^{\prime}\right\rangle\end{array}\right)$ $\frac{1}{\mu}(2 j+1+p)+O(\mu), m=-j-1$

$\left(\begin{array}{c}\left|j, j, m^{\prime}\right\rangle \\ 0\end{array}\right)$
Eigenvalue 
with $\tilde{C}_{1}, \tilde{C}_{2}$ constants satisfying the relation

$$
\frac{\tilde{C}_{1}}{\tilde{C}_{2}}= \begin{cases} \pm 1+O(\mu) & \text { if } p=2 m+1 \\ \frac{1}{\mu}\left(\frac{2 m+1-p \pm|2 m+1-p|}{2 \sqrt{(j-m)(j+m+1)}}\right)+O(\mu) & \text { otherwise. }\end{cases}
$$

Note that

$$
\mathbf{B}_{\mathcal{A}}=\frac{\beta}{4 L} \mathbf{P}_{\mathcal{A}}+O\left(\mu^{2}\right),
$$

hence in the limit $\mu \rightarrow 0$ the eigenvalues of $\mathbf{P}_{\mathcal{A}}$ and $\mathbf{B}_{\mathcal{A}}$ differ by an inessential constant rescaling.

\subsection{Explicit Determination of the Harmonic Spinors}

Let us go back to solving the equation $\not D_{\mathcal{A}} \psi=0$. By Equation (27), writing

$$
\psi=\left(\begin{array}{l}
\Psi \\
\Phi
\end{array}\right)
$$

with $\Psi, \Phi$ 2-component Weyl spinors, the equation $\not D_{\mathcal{A}} \psi=0$ is equivalent to

$$
\mathbf{T}_{\mathcal{A}}^{\dagger} \Phi=0=\mathbf{T}_{\mathcal{A}} \Psi
$$

As shown in Section $4, \mathbf{T}_{\mathcal{A}}^{\dagger}$ has trivial kernel, hence we set $\Phi=0$. Write

$$
\Psi=\left(\begin{array}{l}
K_{1} \\
K_{2}
\end{array}\right) h(r)\left|j, m, m^{\prime}\right\rangle
$$

with $K_{1}, K_{2}$ arbitrary constants, $h$ a radial function to be determined below and $\left|j, m, m^{\prime}\right\rangle$ an eigenvector of the operator $\mathbf{P}_{\mathcal{A}}$ considered in Section 5.1.

Since

$$
\begin{aligned}
\frac{\dot{a}}{a}+\frac{\dot{c}}{2 c} & =\frac{1}{2 L \Lambda \sqrt{V}}\left[-\frac{L V^{\prime}}{2 V}-\operatorname{coth}\left(\frac{r}{2 L}\right)+\frac{3}{2}\left(\frac{(2-\beta) \sinh \left(\frac{r}{2 L}\right)+\beta \cosh \left(\frac{r}{2 L}\right)}{(2-\beta) \cosh \left(\frac{r}{2 L}\right)+\beta \sinh \left(\frac{r}{2 L}\right)}\right)\right], \\
i \mathbf{B}_{\mathcal{A}} & =\frac{1}{4 L \Lambda \sqrt{V}} \frac{i \mathbf{P}_{\mathcal{A}}}{\sinh \left(\frac{r}{2 L}\right)},
\end{aligned}
$$

Equation (63) becomes

$$
\begin{aligned}
\mathbf{T}_{\mathcal{A}}=\frac{i}{2 L \Lambda \sqrt{V}}\left[\left(-2 L \partial_{r}-\frac{L V^{\prime}}{2 V}-\operatorname{coth}\left(\frac{r}{2 L}\right)+\frac{3}{2}\left(\frac{(2-\beta) \sinh \left(\frac{r}{2 L}\right)+\beta \cosh \left(\frac{r}{2 L}\right)}{(2-\beta) \cosh \left(\frac{r}{2 L}\right)+\beta \sinh \left(\frac{r}{2 L}\right)}\right)\right) \mathbf{1}\right. \\
\left.+\frac{\mathbf{P}_{\mathcal{A}}}{2 \sinh \left(\frac{r}{2 L}\right)}\right] .
\end{aligned}
$$


The equation $\mathbf{T}_{\mathcal{A}} \Psi=0$ has non-trivial solutions only for

$$
K_{2}=0, \quad X_{+}\left|j, m, m^{\prime}\right\rangle=0 \quad \Rightarrow \quad m=+j,
$$

or

$$
K_{1}=0, \quad X_{-}\left|j, m, m^{\prime}\right\rangle=0 \quad \Rightarrow \quad m=-j .
$$

If $K_{2}=0, m=j$, substituting in (88) the eigenvalue (76) of $\mathbf{P}_{\mathcal{A}}$, given by

$$
\frac{\lambda}{2}+\frac{2 j+1-\tilde{p}}{\lambda}=\frac{1}{4 L V \sinh \left(\frac{r}{2 L}\right)}+2[(2 j+1) L V-p / \beta] \sinh \left(\frac{r}{2 L}\right),
$$

the equation $\mathbf{T}_{\mathcal{A}} \Psi=0$ reduces to the $\mathrm{ODE}$

$$
\begin{aligned}
-2 L h^{\prime}+ & {\left[-\frac{L V^{\prime}}{2 V}-\operatorname{coth}\left(\frac{r}{2 L}\right)+\frac{3}{2}\left(\frac{(2-\beta) \sinh \left(\frac{r}{2 L}\right)+\beta \cosh \left(\frac{r}{2 L}\right)}{(2-\beta) \cosh \left(\frac{r}{2 L}\right)+\beta \sinh \left(\frac{r}{2 L}\right)}\right)+\right.} \\
& \left.+(2 j+1) L V-\frac{p}{\beta}+\frac{1}{8 L V \sinh ^{2}\left(\frac{r}{2 L}\right)}\right] h=0 .
\end{aligned}
$$

If $K_{1}=0, m=-j$, one obtains the ODE (92) with $p$ replaced by $-p$.

Equation (92) has solution

$$
h=k \frac{[1-\exp (r / L)]^{j}[1+\exp (r / L)-\beta]^{2}}{\left[\exp (2 r / L)-(1-\beta)^{2}\right]^{1 / 2}} \exp \left[-\frac{r}{4 L}\left(3+4 j+\frac{2}{\beta}(p-2 j-1)\right)\right],
$$

with $k$ an arbitrary constant.

For large $r$,

$$
h \simeq \exp \left[-\frac{r}{4 L}(-1+(2 / \beta)(p-2 j-1))\right] .
$$

Since asymptotically $\operatorname{vol}_{\beta} \simeq \exp (-r / L)$, the solution (93) is $L^{2}$ if

$$
2 j+1<p+\beta / 2,
$$

and, as $2 j+1 \geq 1$, there are $L^{2}$ harmonic spinors with $m=j$ only if

$$
p>1-\beta / 2 \geq 0 \Rightarrow p>0 .
$$

For the only other possibility $m=-j$, one obtains instead the condition

$$
2 j+1<-p+\beta / 2,
$$

so there are $L^{2}$ harmonic spinors with $m=-j$ only if

$$
p<-1+\beta / 2 \leq 0 \quad \Rightarrow \quad p<0 .
$$


Given $p \in \mathbb{R}$, the total number of harmonic $L^{2}$ spinors is obtained by summing the multiplicity $2 j+1$, coming from the allowed values for $m^{\prime}$, of the solution (93) over all the allowed values of $j$. Hence

$$
\operatorname{dim}\left(\operatorname{Ker}\left(\mathbf{T}_{\mathcal{A}}\right)\right)=\sum_{2 j+1=1}^{[|p|+\beta / 2]}(2 j+1)=\frac{1}{2}([|p|+\beta / 2])([|p|+\beta / 2]+1),
$$

where $[x]$ is the greatest integer strictly smaller than $x$.

In the TN limit $r=\beta u, \beta \rightarrow 0$, taking $k=(-1)^{j} \beta^{-j+1 / 2} \tilde{k} /(2 \sqrt{2})$ for $\tilde{k}$ a constant,

$$
h \rightarrow \tilde{k} \frac{\rho^{j}}{\sqrt{1+\rho}} \exp \left(\frac{1+2 j-p}{2}\right), \quad \rho=u / L,
$$

so we recover the result in [27].

\subsection{The APS Index Theorem for $M_{\beta}$}

If $(M, g)$ is a Riemannian oriented 4-manifold with boundary $\partial M$ and $\not D_{\mathcal{A}}$ is the Dirac operator twisted by a connection $\mathcal{A}$ with curvature $\mathcal{F}$, by the Atiyah-Patodi-Singer (APS) index theorem, see e.g. [13],

$$
\operatorname{index}\left(\not D_{\mathcal{A}}\right)=\frac{1}{192 \pi^{2}} \int_{M} \operatorname{Tr}\left(\Omega^{2}\right)+\frac{1}{8 \pi^{2}} \int_{M} \mathcal{F} \wedge \mathcal{F}-\frac{1}{192 \pi^{2}} \int_{\partial M} \operatorname{Tr}(\theta \wedge \Omega)-\frac{1}{2}(\eta(0)+h) \text {. }
$$

Here $\Omega$ is the curvature of some connection on $M, \operatorname{Tr}\left(\Omega^{2}\right)=-\Omega_{a b} \wedge \Omega_{a b}, \theta$ is the second fundamental form of $\partial M$ and $\eta(0)+h$ is a non-local boundary contribution depending on the spectrum of the boundary Dirac operator. The first two terms are the usual bulk contributions to the index, the third one is a local boundary contribution only arising if $g$ is not a product metric in a neighbourhood of $\partial M$ [19]. The $\eta$-invariant is further discussed in Appendix A. The operator $\not D_{\mathcal{A}}$ in (101) acts on $L^{2}$ spinors satisfying certain global boundary conditions to be discussed below.

In order to make use of (101), we consider the truncation

$$
\bar{M}_{\beta, r_{0}}=\left\{(r, \theta, \psi, \phi) \in M_{\beta}: r \leq r_{0}\right\}
$$

of $M_{\beta}$ at some finite radius $r_{0}$, a manifold with boundary

$$
\partial \bar{M}_{\beta, r_{0}}=\left\{(r, \theta, \psi, \phi) \in M_{\beta}: r=r_{0}\right\},
$$

and then take the limit $r_{0} \rightarrow \infty$.

Taking $\mathcal{F}=\mathrm{d} \mathcal{A}, \mathcal{A}$ given by (62), the volume element (49) and integrating from $\infty$ to 0 in radial integrals, for the bulk contributions we obtain

$$
\begin{gathered}
-\frac{1}{2} \int_{M_{\beta}} \operatorname{Tr}\left(\Omega^{2}\right)=4 \pi^{2}\left(2+\beta^{2}\right), \\
\int_{M_{\beta}} \mathcal{F} \wedge \mathcal{F}=4 \pi^{2} p^{2},
\end{gathered}
$$


where to compute (104) one can use e.g. the curvature form (9) with $b=a, B=A$ and the values $a, c, f, A, C$ of $g_{\beta}$ given by (66).

Let $g^{\prime}$ be a Riemannian metric on $M$ which is a product metric in a neighbourhood of $\partial \bar{M}_{\beta, r_{0}}$ and which agrees with $g$ on $\partial \bar{M}_{\beta, r_{0}}, \omega^{\prime}$ the Levi-Civita connection associated to $g^{\prime}$. The 1 -form $\theta$ can be computed as the difference [13]

$$
\theta=\omega-\omega^{\prime}
$$

We take

$$
g^{\prime}=\Lambda^{2}\left(r_{0}\right)\left[a_{\mathrm{hTN}}^{2}\left(r_{0}\right)\left(\eta_{1}^{2}+\eta_{2}^{2}\right)+c_{\mathrm{hTN}}^{2}\left(r_{0}\right) \eta_{3}^{2}+f_{\mathrm{hTN}}^{2}\left(r_{0}\right) \mathrm{d} r^{2}\right] .
$$

After some computations, we find that the only non-vanishing components of $\theta$ are

$$
\begin{aligned}
\theta_{i 4} & =-\frac{\exp (r / 2 L)\left(4-5 \beta+\beta^{2}+\exp (r / L)(-4+3 \beta)\right) \eta_{i}}{2\left(\exp (2 r / L)-(\beta-1)^{2}\right)}, i=1,2, \\
\theta_{34} & =-\beta \frac{\left(\exp (3 r / L)+\exp (2 r / L)(\beta-3)+\exp (r / L)(\beta-1)(-3+2 \beta)-(\beta-1)^{2}\right) \eta_{3}}{2\left(\exp (2 r / L)-(\beta-1)^{2}\right)(\exp (r / L)+\beta-1)} .
\end{aligned}
$$

The contribution from the boundary integral is then

$$
\lim _{r_{0} \rightarrow \infty} \int_{\partial \bar{M}_{\beta, r_{0}}} \operatorname{Tr}(\theta \wedge \Omega)=-8 \pi^{2} \beta^{2} .
$$

The $\eta$-invariant only depends on the geometry of a large $r$ hypersurface, and is unaffected by a constant rescaling of the metric. By Equation (47), the metric induced by $g_{\beta}$ on a large $r$ hypersurface is

$$
\frac{4 L^{2}}{\beta^{2}}\left(\eta_{1}^{2}+\eta_{2}^{2}+\beta^{2} \exp (-r / L) \eta_{3}^{2}\right)
$$

hence the geometry is that of a squashed 3-sphere. The $\eta$-invariant for this geometry has been considered in [23] for the non-twisted Dirac operator, and in [34] for the twisted one. The result, which for completeness is also derived in Appendix A, is

$$
\eta(0)+h=-\frac{1}{6}+p^{2}-[|p|]([|p|]+1) .
$$

Substituting (104), (105), (109), (111) in (101) we get, since $\operatorname{Ker}\left(\mathbf{T}_{\mathcal{A}}^{\dagger}\right)=0$,

$$
\operatorname{index}\left(\not D_{\mathcal{A}}\right)=\operatorname{dim}\left(\operatorname{Ker}\left(\mathbf{T}_{\mathcal{A}}\right)\right)-\operatorname{dim}\left(\operatorname{Ker}\left(\mathbf{T}_{\mathcal{A}}^{\dagger}\right)\right)=\operatorname{dim}\left(\operatorname{Ker}\left(\mathbf{T}_{\mathcal{A}}\right)\right)=\frac{1}{2}[|p|](\mid[p \mid]+1)
$$

Apparently, for $\beta \neq 0$ (112) is in disagreement with the result (99) obtained by counting the explicit solutions. However, the disagreement only arises because we have 
not yet taken into account the boundary conditions of the APS index theorem. In fact, in $(101) \not D_{\mathcal{A}}$ acts on $L^{2}$ spinors subject to the non-local boundary conditions

$$
\begin{aligned}
& \left\langle\left\langle v_{z}, \Psi\right\rangle\right\rangle=0 \quad \forall z \geq 0, \\
& \left\langle\left\langle v_{z}, \Phi\right\rangle\right\rangle=0 \quad \forall z<0,
\end{aligned}
$$

where $(\Psi, \Phi)^{T}$ is an $L^{2}$ harmonic spinor for the operator $\not D_{\mathcal{A}}$ on $M_{\beta}, v_{z}$ is an eigenvector of the boundary Dirac operator $\mathbf{P}_{\mathcal{A}}$ with eigenvalue $z$, and

$$
\left\langle\left\langle v_{z}, \Psi\right\rangle\right\rangle=\lim _{r_{0} \rightarrow \infty} \int_{\partial \bar{M}_{\beta, r_{0}}} v_{z}^{\dagger} \Psi \operatorname{vol}_{\partial \bar{M}_{\beta, r_{0}}} .
$$

As shown in Section 5.2, harmonic spinors are of the form $(\Psi, 0)^{T}$, with $\Psi \propto$ $\left(\left|j, j, m^{\prime}\right\rangle, 0\right)^{T}$ if $p>0, \Psi \propto\left(0,\left|j,-j, m^{\prime}\right\rangle\right)^{T}$ if $p<0$. Hence, (114) automatically holds, but we need to impose (113). Since $\left\langle j_{1}, m_{1}, m_{1}^{\prime} \mid j_{2}, m_{2}, m_{2}^{\prime}\right\rangle \propto \delta_{j_{1} j_{2}} \delta_{m_{1} m_{2}} \delta_{m_{1}^{\prime} m_{2}^{\prime}}$, if $p>0$ the only non-trivial condition is the orthogonality of $\Psi$ to the asymptotic eigenvector $\left(\left|j, j, m^{\prime}\right\rangle, 0\right)^{T}$ of $\mathbf{P}_{\mathcal{A}}$, see (81), when the corresponding eigenvalue is non-negative. This is the case for

$$
2 j+1-p \geq 0 .
$$

Similarly, if $p<0$ we only need to impose the orthogonality of $\Psi$ to the asymptotic eigenvector $\left(0,\left|j,-j, m^{\prime}\right\rangle\right)^{T}$ of $\mathbf{P}_{\mathcal{A}}$ when the corresponding eigenvalue is non-negative, that is, see $(80)$, for

$$
2 j+1+p \geq 0 .
$$

Therefore, the APS index theorem only counts $L^{2}$ harmonic spinors for which $2 j+1<$ $|p|$, in agreement with explicit counting of the solutions satisfying the same condition, given by (99) with the sum only extending up to $[|p|]$.

For $\beta \in(0,2]$, we can consider if our harmonic spinors extend to the compactification $P_{\beta}^{2}(\mathbb{C})$ of $M_{\beta}$, which has the topology of $P^{2}(\mathbb{C})$. The topology is now non-trivial, which results in a quantisation condition on the spinor charge $p[21]$,

$$
p=k+\frac{1}{2}, \quad k \in \mathbb{Z} .
$$

Requiring the harmonic spinors $(93)$ to extend to $P_{\beta}^{2}(\mathbb{C})$, i.e. to have a finite limit as $r \rightarrow \infty$, we get the condition

$$
2 j+1 \leq p-\beta / 2=k+(1-\beta) / 2 .
$$

The number of harmonic spinors extending to the compactified manifold is therefore

$$
\sum_{2 j+1=1}^{\lfloor k+(1-\beta) / 2\rfloor}(2 j+1)= \begin{cases}k(k+1) / 2 & \text { if } \beta \in(0,1], \\ k(k-1) / 2 & \text { if } \beta \in(1,2],\end{cases}
$$

where $\lfloor\cdot\rfloor$ is the floor function. 
For $\beta=1, P_{\beta}^{2}(\mathbb{C})$ is $P^{2}(\mathbb{C})$ with the FS metric. The index theorem for the twisted Dirac operator on a closed manifold can be obtained from (101) by dropping the boundary terms. Using (104), (105) with $\beta=1, p=k+1 / 2$, we have

$$
\frac{1}{192 \pi^{2}} \int_{P^{2}(\mathbb{C})} \operatorname{Tr}\left(\Omega^{2}\right)+\frac{1}{8 \pi^{2}} \int_{P^{2}(\mathbb{C})} \mathcal{F} \wedge \mathcal{F}=-\frac{1}{8}+\frac{1}{2}\left(k+\frac{1}{2}\right)^{2}=\frac{k(k+1)}{2},
$$

in agreement with (120), see also [21].

The isometry group of $P^{2}(\mathbb{C})$ is $S U(3)$, with $S U(3) / \mathbb{Z}_{3}$ acting effectively [18]. Finite dimensional irreducible representations of $S U(3)$ are classified by pairs $\left(m_{1}, m_{2}\right)$ of nonnegative integers, with the representation $\left(m_{1}, m_{2}\right)$ having dimension

$$
\left(m_{1}+1\right)\left(m_{2}+1\right)\left(m_{1}+m_{2}+2\right) / 2 .
$$

The group $S U(3)$ has $S U(2) \times U(1)$ as a subgroup and harmonic spinors on $P^{2}(\mathbb{C})$ of charge $p=k+1 / 2, k \in \mathbb{Z}$, fall into the $S U(3)$ representation $(k-1,0)$ if $p \geq 3 / 2$, the representation $(0,|k|-2)$ if $p \leq-3 / 2$. A detailed study of the spectrum of the twisted Dirac operator on $P^{2}(\mathbb{C})$ can be found in [12].

For $\beta \neq 1, P_{\beta}^{2}(\mathbb{C})$ has an edge cone singularity of cone angle $2 \pi \beta$ along $S_{\infty}^{2}$, hence the index theorem for closed manifolds needs to be modified to take into account the conical singularity. Such an extension has been considered in [4], where the following result is proved: Let $X$ be a spin oriented 4-manifold, $Y$ be a smooth compact oriented embedded surface, $g$ an incomplete edge metric on $X \backslash Y$ with cone angle $2 \pi \beta$ along $Y$. Then, for $\beta \in(0,1]$,

$$
\operatorname{index}(\not D)=\frac{1}{192 \pi^{2}} \int_{X} \operatorname{Tr}\left(\Omega^{2}\right)+\frac{1}{24}\left(\beta^{2}-1\right)[Y]^{2},
$$

where $[Y]$ is the self-intersection number of $Y$ in $X$.

Since $P_{\beta}^{2}(\mathbb{C})$ is not spin, $(123)$ does not apply. Nevertheless, let us take $X=P_{\beta}^{2}(\mathbb{C})$, $Y=S_{\infty}^{2}$ and calculate the obvious extension of (123) to a twisted Dirac complex,

$$
\frac{1}{192 \pi^{2}} \int_{P_{\beta}^{2}(\mathbb{C})} \operatorname{Tr}\left(\Omega^{2}\right)+\frac{1}{8 \pi^{2}} \int_{P_{\beta}^{2}(\mathbb{C})} \mathcal{F} \wedge \mathcal{F}+\frac{1}{24}\left(\beta^{2}-1\right)\left[S_{\infty}^{2}\right]^{2}=\frac{k(k+1)}{2}
$$

having used $\left[S_{\infty}^{2}\right]^{2}=1,(104)$ and (105) with $p=k+1 / 2$. The result is in agreement with (120) suggesting that, at least in this case, the extension of (123) to a twisted Dirac operator is indeed given by (124). Note that (123) requires $\beta \in(0,1]$, in which case a particular geometric Witt condition holds [4], and in fact (124) and (120) only agree for $\beta$ in this range. 


\section{Conclusions}

The main contribution of this paper is the explicit determination of all the harmonic spinors on the 1-parameter family of Einstein metrics $g_{\beta}, \beta \in[0,2]$, defined on a manifold with the topology of $\mathbb{C}^{2}$. Because of the high degree of symmetry of the problem, it has been possible to describe harmonic spinors as eigenvectors of the twisted Dirac operator on the squashed 3-sphere $\mathbf{P}_{\mathcal{A}}$, with a radial part obeying the ODE (92).

The kernel of the non-twisted Dirac operator is trivial, but once the Dirac operator is coupled to the geometrically preferred connection $\mathcal{A}(62)$ of strength $p \in \mathbb{R}$, its kernel decomposes as the direct sum of the irreducible $S U(2)$ representations of dimension up to $[p+\beta / 2]$. A very similar behaviour is exhibited by harmonic spinors on the TN manifold [27, 29], which are in fact recovered as the $\beta \rightarrow 0$ limit of our solution (93). For the special values $0,1,2$ of the parameter $\beta$, the curvature $\mathcal{F}=\mathrm{d} \mathcal{A}$ is the unique harmonic $L^{2}$ form on $M_{\beta}$. It would be interesting to know if such uniqueness holds for all values of $\beta$.

We have compared the index of $D_{\mathcal{A}}$ as obtained by direct counting of the solutions with the value obtained via the Atiyah-Patodi-Singer (APS) index theorem. Of course, the two results have to agree, but to show that they do the subtle boundary conditions of the APS index theorem need to be taken into full account - this is often not the case as in typical applications to non-compact manifolds, the spaces under consideration have infinite volume and the APS boundary conditions are trivially satisfied by $L^{2}$ spinors.

We have also considered the extension of our solutions to the compactification $P_{\beta}^{2}(\mathbb{C})$ of $M_{\beta}$, a topologically non-trivial manifold homeomorphic to $P^{2}(\mathbb{C})$. For $\beta=1, P_{\beta}^{2}(\mathbb{C})$ is $P^{2}(\mathbb{C})$ with the smooth FS metric and our results are in agreement with those in the literature. For all the other values of $\beta$ in $(0,2)$, the metric $g_{\beta}$ on $M_{\beta}$ extends to $P_{\beta}^{2}(\mathbb{C})$ as an edge-cone metric and our analysis contributes to the understanding of the Dirac operator in this type of geometries.

\section{Acknowledgements}

The author would like to thank Derek Harland and Bernd Schroers for useful discussions, and acknowledge support by the DFG Research Training Group No. 1463. 


\section{A The $\eta$-invariant}

Let $M$ be a Riemannian 4-manifold with boundary, $D_{\mathcal{A}}$ the Dirac operator on $M$ twisted by a connection $\mathcal{A}, \mathbf{P}_{\mathcal{A}}$ the Dirac operator induced on $\partial M$. The $\eta$-invariant is the analytic continuation to $s=0$ of the meromorphic function $\eta(s)$ defined for $\Re(s)>2$ by

$$
\eta(s)=\sum_{z>0} \frac{1}{z^{s}}-\sum_{z<0} \frac{1}{(-z)^{s}},
$$

where the sum is taken over the non-zero eigenvalues $z$ of the boundary Dirac operator $\mathbf{P}_{\mathcal{A}}[8,34]$. We also define

$$
h=\operatorname{dim}\left(\operatorname{Ker}\left(\mathbf{P}_{\mathcal{A}}\right)\right) .
$$

In our case, $M=\bar{M}_{\beta, r_{0}}$ and the eigenvalues of $\mathbf{P}_{\mathcal{A}}$ are given by (79)-(81). The eigenvalues (79) are non-vanishing and come in pairs of elements with opposite sign, thus giving a net zero contribution to the $\eta$-invariant. The non-vanishing contribution comes from (80), (81). The $\eta$-invariant is unchanged under a constant rescaling of all the eigenvalues, hence we can multiply (80), (81) by $\mu$ before taking the limit $\mu \rightarrow 0$, obtaining

$$
\begin{cases}2 j+1-p & \text { if } m=j, \\ 2 j+1+p & \text { if } m=-j-1\end{cases}
$$

Writing $d$ for the multiplicity $2 j+1$ of each eigenvalue, and assuming for notational simplicity $p>0$ (the case $p<0$ is dealt with by replacing $p$ with $|p|$ ), we have

$$
\begin{aligned}
\eta(s) & =\sum_{d=1}^{\infty} \frac{d}{(d+p)^{s}}+\sum_{\substack{d=[p]+1 \\
d \neq p}}^{\infty} \frac{d}{(d-p)^{s}}-\sum_{d=1}^{[p]} \frac{d}{(p-d)^{s}} \\
& =\sum_{d=1}^{\infty} \frac{d}{(d+p)^{s}}+\sum_{\substack{d=1 \\
d \neq p}}^{\infty} \frac{d}{(d-p)^{s}}-\sum_{d=1}^{[p]} \frac{d}{(d-p)^{s}}-\sum_{d=1}^{[p]} \frac{d}{(p-d)^{s}} .
\end{aligned}
$$

The condition $d \neq p$ is trivially satisfied for $p \notin \mathbb{N}$, that is $\chi_{\mathbb{N}}(p)=0$ with $\chi_{\mathbb{N}}(p)$ the characteristic function of $\mathbb{N}$. The last two sums are finite and their value for $s=0$ is

$$
-2 \sum_{d=1}^{[p]} d=-[p]([p]+1) .
$$

We rewrite the first two terms of (128) as follows,

$$
\begin{aligned}
& \sum_{d=1}^{\infty} \frac{d}{(d+p)^{s}}=\sum_{d=1}^{\infty} \frac{1}{d^{s-1}}\left(1+\frac{p}{d}\right)^{-s}=\sum_{d=1}^{\infty} \frac{1}{d^{s-1}}\left(1-s \frac{p}{d}+\frac{1}{2} s(s+1)\left(\frac{p}{d}\right)^{2}+\cdots\right)= \\
& =\zeta(s-1)-\operatorname{sp} \zeta(s)+\frac{1}{2} s(s+1) p^{2} \zeta(s+1)+\cdots
\end{aligned}
$$




$$
\begin{aligned}
& \sum_{\substack{d=1 \\
d \neq p}}^{\infty} \frac{d}{(d-p)^{s}}=\sum_{\substack{d=1 \\
d \neq p}}^{\infty} \frac{1}{d^{s-1}}\left(1+s \frac{p}{d}+\frac{1}{2} s(s+1)\left(\frac{p}{d}\right)^{2}+\cdots\right)=\zeta(s-1)-\chi_{\mathbb{N}}(p) p^{1-s}+ \\
& +s p\left[\zeta(s)-\chi_{\mathbb{N}}(p) p^{-s}\right]+\frac{1}{2} s(s+1) p^{2}\left[\zeta(s+1)-\chi_{\mathbb{N}}(p) p^{-(s+1)}\right]+\cdots
\end{aligned}
$$

where $\zeta$ is the Riemann zeta function. Summing (130) and (131) gives

$$
2 \zeta(s-1)-\chi_{\mathbb{N}}(p) p^{1-s}+s(s+1) p^{2} \zeta(s+1)+\cdots \stackrel{s \rightarrow 0}{\longrightarrow}-\frac{1}{6}+p^{2}-\chi_{\mathbb{N}}(p) p,
$$

as $\zeta(-1)=-1 / 12, \lim _{s \rightarrow 0} s \zeta(s+1)=1$, and all the omitted terms in (132) vanish in the limit $s \rightarrow 0$.

From (127) we see that, for $p>0, \mathbf{P}_{\mathcal{A}}$ has zero as an eigenvalue if and only if $m=j$, $p=2 j+1 \in \mathbb{N}$, in which case the eigenvalue multiplicity is $2 j+1=p$. Hence $h=\chi_{\mathbb{N}}(p) p$. An entirely similar result holds for $p<0$, hence for any $p \in \mathbb{R} \backslash\{0\}$

$$
\eta(0)+h=-\frac{1}{6}+p^{2}-[|p|]([|p|]+1) .
$$

\section{B Left- and Right-invariant Vector Fields on $S U(2)$}

For convenience, we summarise here some facts about left- and right-invariant 1-forms and vector fields on $S U(2)$. We follow the conventions in [27]. Let $z_{1}, z_{2}$ denote complex coordinates in $\mathbb{C}^{2}$. An element $h \in S U(2)$ can be written as

$$
h=\left(\begin{array}{cc}
z_{1} & -\bar{z}_{2} \\
z_{2} & \overline{z_{1}}
\end{array}\right)
$$

with the constraint $\left|z_{1}\right|^{2}+\left|z_{2}\right|^{2}=1$. In terms of the Euler angles $\theta \in[0, \pi], \phi \in[0,2 \pi)$, $\psi \in[0,4 \pi)$ we can write

$$
\begin{aligned}
& z_{1}=\cos \left(\frac{\theta}{2}\right) \mathrm{e}^{-\frac{i}{2}(\psi+\phi)}, \\
& z_{2}=\sin \left(\frac{\theta}{2}\right) \mathrm{e}^{-\frac{i}{2}(\psi-\phi)}
\end{aligned}
$$

We take the basis $\left\{t_{1}, t_{2}, t_{3}\right\}$ of $\mathfrak{s u}(2)$, with $t_{i}=-\frac{i}{2} \boldsymbol{\sigma}_{i}, \boldsymbol{\sigma}_{i}$ the $i$-th Pauli matrix. The chosen basis is orthonormal with respect to the product $\langle A, B\rangle_{\mathfrak{s u}(2)}=-2 \operatorname{Tr}(A B)$. Note that $h$ can be written as

$$
h=\mathrm{e}^{t_{3} \phi} \mathrm{e}^{t_{2} \theta} \mathrm{e}^{t_{3} \psi} .
$$

We can decompose the left-invariant Maurer-Cartan 1-form $\Theta$ as

$$
\Theta=h^{-1} \mathrm{~d} h=\eta_{1} t_{1}+\eta_{2} t_{2}+\eta_{3} t_{3} .
$$


The left-invariant 1-forms $\left\{\eta_{i}\right\}$ are then given by

$$
\begin{aligned}
& \eta_{1}=\sin \psi \mathrm{d} \theta-\cos \psi \sin \theta \mathrm{d} \phi \\
& \eta_{2}=\cos \psi \mathrm{d} \theta+\sin \psi \sin \theta \mathrm{d} \phi, \\
& \eta_{3}=\mathrm{d} \psi+\cos \theta \mathrm{d} \phi .
\end{aligned}
$$

They satisfy the relation

$$
\mathrm{d} \eta_{i}=-\frac{1}{2} \epsilon_{i j k} \eta_{j} \wedge \eta_{k}
$$

The dual left-invariant vector fields are

$$
\begin{aligned}
& X_{1}=\sin \psi \partial_{\theta}+\frac{\cos \psi}{\sin \theta}\left(\cos \theta \partial_{\psi}-\partial_{\phi}\right), \\
& X_{2}=\cos \psi \partial_{\theta}-\frac{\sin \psi}{\sin \theta}\left(\cos \theta \partial_{\psi}-\partial_{\phi}\right), \\
& X_{3}=\partial_{\psi} .
\end{aligned}
$$

Similarly for the right-invariant Maurer-Cartan 1-form $\zeta$ we have

$$
\zeta=\mathrm{d} h h^{-1}=\zeta_{1} t_{1}+\zeta_{2} t_{2}+\zeta_{3} t_{3},
$$

with

$$
\begin{aligned}
& \zeta_{1}=\cos \phi \sin \theta \mathrm{d} \psi-\sin \phi \mathrm{d} \theta, \\
& \zeta_{2}=\sin \phi \sin \theta \mathrm{d} \psi+\cos \phi \mathrm{d} \theta, \\
& \zeta_{3}=\mathrm{d} \phi+\cos \theta \mathrm{d} \psi .
\end{aligned}
$$

The dual right-invariant vector fields are

$$
\begin{aligned}
& Z_{1}=-\sin \phi \partial_{\theta}-\frac{\cos \phi}{\sin \theta}\left(\cos \theta \partial_{\phi}-\partial_{\psi}\right), \\
& Z_{2}=\cos \phi \partial_{\theta}-\frac{\sin \phi}{\sin \theta}\left(\cos \theta \partial_{\phi}-\partial_{\psi}\right), \\
& Z_{3}=\partial_{\phi} .
\end{aligned}
$$

Note that the transformation $(\psi, \theta, \phi) \mapsto(-\phi,-\theta,-\psi)$ maps $\left(X_{i}, \eta_{i}\right) \rightarrow\left(-Z_{i},-\zeta_{i}\right)$. 


\section{References}

[1] M. Abreu. "Kähler metrics on toric orbifolds". In: J. Diff. Geom. 58 (2001), pp. 151-187.

[2] O. Aharony, S. S. Razamat, N. Seiberg, and B. Willett. "3d dualities from 4d dualities". In: J. High Energy Phys. 07 (2013), p. 149.

[3] O. Aharony et al. "Aspects of $N=2$ supersymmetric gauge theories in three dimensions". In: Nucl. Phys. B 499 (1997), pp. 67-99.

[4] P. Albin and J. Gell-Redman. "The index of Dirac operators on incomplete edge spaces". In: SIGMA 12 (2016), p. 45.

[5] B. Amman, M. Dahl, and E. Humbert. "Surgery and harmonic spinors". In: Adv. Math. 220 (2009), pp. 523-539.

[6] M. F. Atiyah and C. Lebrun. "Curvature, cones, and characteristic numbers". In: Math. Proc. Cambridge Philos. Soc. 155 (2012), pp. 13-37.

[7] M. F. Atiyah, N. S. Manton, and B. J. Schroers. "Geometric models of matter". In: Proc. Roy. Soc. Lond. A 468 (2012), pp. 1252-1279.

[8] M. F. Atiyah, V. K. Patodi, and I. M. Singer. "Spectral asymmetry and Riemannian geometry. I". In: Math. Proc. Camb. Phil. Soc. 77 (1975), p. 43.

[9] C. Bär. "Metrics with harmonic spinors". In: Geom. Funct. Anal. 6 (1996), pp. 899 942.

[10] C. Bär and P. Schmutz. "Harmonic spinors on Riemann surfaces". In: Ann. Global Anal. Geom. 10 (1992), pp. 263-273.

[11] S. A. Cherkis and A. Kapustin. "Hyper-Kähler metrics from periodic monopoles". In: Phys. Rev. D 65 (2002), p. 084015.

[12] B. P. Dolan and R. J. Szabo. "Dimensional reduction and vacuum structure of quiver gauge theory". In: J. High Energy Phys. 08 (2009), p. 038.

[13] T. Eguchi, P. B. Gilkey, and A. J. Hanson. "Gravitation, gauge theories and differential geometry". In: Phys. Rep. 66 (1980), pp. 213-393.

[14] G. Franchetti. "Harmonic forms on ALF gravitational instantons". In: J. High Energy Phys. 12 (2014), p. 075.

[15] G. Franchetti and N. S. Manton. "Gravitational instantons as models for charged particle systems". In: J. High Energy Phys. 03 (2013), p. 072.

[16] G. W. Gibbons and S. W. Hawking. "Classification of gravitational instanton symmetries". In: Commun. Math. Phys. 66 (1979), pp. 291-310.

[17] G. W. Gibbons and S. W. Hawking. "Gravitational multi-instantons". In: Phys. Lett. B 78 (1978), pp. 430-432.

[18] G. W. Gibbons and C. N. Pope. "CCP $P^{2}$ as a gravitational instanton". In: Comm. Math. Phys. 61 (1978), pp. 239-248. 
[19] P. B. Gilkey. "On the index of geometrical operators for Riemannian manifolds with boundary". In: Adv. Math. 102 (1993), pp. 129-183.

[20] T. Hausel, E. Hunsicker, and R. Mazzeo. "Hodge cohomology of gravitational instantons". In: Duke Math. J. 122 (2004), pp. 485-548.

[21] S. W. Hawking and C. N. Pope. "Generalized spin structures in quantum gravity". In: Phys. Lett. B 73 (1978), pp. 42-44.

[22] N. J. Hitchin. "A new family of Einstein metrics". In: Manifolds and Geometry (Pisa 1993). Ed. by P. de Bartolomeis, F. Tricerri, and E. Vesentini. Vol. XXXVI. Sympos. Math. Cambridge University Press, 1996, pp. 190-222.

[23] N. J. Hitchin. "Harmonic spinors". In: Adv. Math. 14 (1974), pp. 1-55.

[24] N. J. Hitchin. " $L^{2}$-cohomology of hyperkähler quotients". In: Commun. Math. Phys. 211 (2000), pp. 153-165.

[25] N. J. Hitchin and M. F. Atiyah. The Geometry and Dynamics of Magnetic Monopoles. Princeton University Press, 2014.

[26] R. Jackiw and C. Rebbi. "Solitons with fermion number $\frac{1}{2}$ ". In: Phys. Rev. D 13 (1976), pp. 3398-3409.

[27] R. Jante and B. J. Schroers. "Dirac operators on the Taub-NUT space, monopoles and SU(2) representations". In: J. High Energy Phys. 1401 (2014), p. 114.

[28] R. Jante and B. J. Schroers. "Spectral properties of Schwarzschild instantons". In: Class. Quantum Grav. 33 (2016), p. 205008.

[29] R. Jante and B. J. Schroers. "Taub-NUT dynamics with a magnetic field". In: $J$. Geometry Phys. 104 (2016), pp. 305-328.

[30] H. B. Lawson and M. L. Michelsohn. Spin geometry. Princeton University Press, 1989.

[31] C. LeBrun. "Explicit self-dual metrics on $C P^{2} \# \ldots \# C P^{2}$ ". In: J. Diff. Geom. 34 (1991), pp. 223-253.

[32] K. Peeters and A. K. Waldron. "Spinors on manifolds with boundary: APS index theorems with torsion". In: J. High Energy Phys. 02 (1999), p. 024.

[33] C. N. Pope. "Axial-vector anomalies and the index theorem in charged Schwarzschild and Taub-NUT spaces". In: Nucl. Phys. B 141 (1978), pp. 432-444.

[34] C. N. Pope. "The $\eta$ invariant for charged spinors in Taub-NUT". In: J. Phys. A 14 (1981), pp. L133-L137.

[35] N. Seiberg and E. Witten. "Gauge dynamics and compactification to three dimensions". In: The Mathematical Beauty of Physics: A Memorial Volume for Claude Itzykson. Saclay: World Scientific, 1996, pp. 333-366.

[36] A. Sen. "Dyon-monopole bound states, self-dual harmonic forms on the multimonopole moduli space, and $S L(2, Z)$ invariance in string theory". In: Phys. Lett. B 329 (1994), pp. 217-221. 
[37] Edward Witten. "Cosmic superstrings". In: Phys. Lett. B 153 (1985), pp. 243-246. 